Two coefficients deserve special mention. One is $\sigma_{4}$ for $\alpha=90^{\circ}$, which is equal to $(4 / 15)\left[K\left(\sin 45^{\circ}\right)\right]^{4}$; the other is $\sigma_{6}$ for $\alpha=60^{\circ}$ or $120^{\circ}$, which is equal to $(64 \sqrt{ } 3 / 315)\left[K\left(\sin 15^{\circ}\right)\right]^{6}$, where $K(k)$ represents the complete elliptic integral of the first kind in Legendre form, of modulus $k$. The values of these coefficients to $25 \mathrm{D}$ are:

$$
\begin{aligned}
& \sigma_{4}=3.1512120021538975382176899 \text {, } \\
& \sigma_{6}=5.8630316934254015979702134 .
\end{aligned}
$$

For the convenience of the reader, there is included in Table 3 a compilation of $25 \mathrm{D}$ values of the tangent and cotangent for arguments $1^{\circ}\left(1^{\circ}\right) 89^{\circ}$. These data, which are required in computing the values of $c$, are here tabulated with the same range and precision as for the values of sine and cosine given by G. W. and R. M. Spenceley [4]. The only comparable table of decimal approximations to the tangent appears to be the relatively inaccessible 30D table of Herrmann [5].

Virginia Polytechnic Institute

Blacksburg, Virginia

1. C. B. Ling, "Evaluation at half periods of Weierstrass' elliptic function with rectangular primitive period-parallelogram," Math. Comp., v. 14, 1960, pp. 67-70. MR 22 *1061.

2. C. B. LiNG \& C. P. TSAI, "Evaluation at half periods of Weierstrass' elliptic function with rhombic primitive period-parallelogram," Math. Comp., v. 18, 1964, pp. 433-440. (Erratum: A negative sign should precede $K_{3}$ in the first of Eqs. (7) on p. 434.)

3. C. B. Ling, "Tables of values of $\sigma_{2 s}$ relating to Weierstrass' elliptic function," Math. Comp., v. 19, 1965, pp. 123-127.

4.'G. W. \& R. M. SPENCELEY, Smithsonian Elliptic Function Tables, Smithsonian Miscellaneous Collections, Vol. 109, Smithsonian Institution, Washington, D. C., 1947, p. 366. MR 9, 380 .

5. HerrmanN, "Bestimmung der trigonometrischen Functionen aus den Winkeln und der Winkel aus den Functionen, bis zu einer beliebigen Grenze der Genauigkeit," Kaiserliche Akademic der Wissenschaften, Wien, Math.-Naturwiss. Classe, Sitzungsberichte, v. 1, 1848, part IV, pp. 178-179.

\title{
The Asymptotic Expansion of the Integrals Psi and Chi In Terms of Tchebycheff Polynomials
}

\author{
By G. T. Thompson
}

Hummer [3] has given the expansion of the Dawson function which is used in the calculation of line-absorbtion coefficient due to Doppler effect and damping. Psi and Chi are two integrals used to determine the Doppler broading effect on neutron cross sections in the resonance region [1]. They are functions of two variables and are given by

$$
\begin{aligned}
& \psi(x, \theta)=\frac{1}{\sqrt{ } 4 \theta} \int_{-\infty}^{\infty} \frac{\exp \left[-(y-x)^{2} / 4 \theta\right]}{1+y^{2}} d y \\
& \chi(x, \theta)=\frac{1}{\sqrt{ } 4 \theta} \int_{-\infty}^{\infty} \frac{y \exp \left[-(y-x)^{2} / 4 \theta\right]}{1+y^{2}} d y
\end{aligned}
$$

and satisfy these conditions

Received February 4, 1965. 


$$
0<\psi(x, \theta) \leqq 1 ; \quad \psi(-x, \theta)=\psi(x, \theta)
$$

and

$$
-1<\chi(x, \theta)<1 ; \quad \chi(-x, \theta)=-\chi(x, \theta) .
$$

Chi is related to Psi by the formula

$$
\chi=2 \theta \frac{\partial \psi}{\partial x}+x \psi
$$

Both Psi and Chi can be expanded in a uniformly convergent series in terms of the incomplete gamma function. By making a change of the variable of integration, we have

$$
\begin{aligned}
\psi(x, \theta) & =\exp \left[-\left(x^{2}-1\right) / 4 \theta\right](4 \theta)^{-1 / 2} \int_{1 / 4 \theta}^{\infty} u^{-1 / 2} e^{-u} \exp \left[\frac{-1}{u}\left(\frac{x}{4 \theta}\right)^{2}\right] d u \\
& =(4 \theta)^{-1 / 2} \exp \left[-\left(x^{2}-1\right) / 4 \theta\right] \int_{1 / 4 \theta}^{\infty} e^{-u} u^{-1 / 2} \sum_{i=0}^{\infty} \frac{1}{i !}\left[\frac{1}{u}\left(\frac{x}{4 \theta}\right)^{2}\right] d u .
\end{aligned}
$$

Let

$$
\Gamma_{i}(\theta)=\frac{e^{-1 / 4 \theta}}{\sqrt{ } 4 \theta} \int_{1 / 4 \theta}^{\infty} u^{(-2 i+1) / 2} e^{-u} d u
$$

Then

$$
\Gamma_{i+1}(\theta)=\frac{2}{2 i+1}\left[(4 \theta)^{i}-\Gamma_{i}(\theta)\right]
$$

and

$$
\psi(x, \theta)=e^{-x^{2} / 4 \theta} \sum_{i=0}^{\infty} \frac{1}{i !}\left(\frac{x}{4 \theta}\right) 2 i \Gamma_{i}(\theta) .
$$

The incomplete gamma function is $e^{1 / 4 \theta}$ times $\Gamma_{0}(\theta)$. The term by term integration is justified since the initial series converges uniformly. Similarly

$$
\chi(x, \theta)=e^{-x^{2} / 4 \theta} \sum_{k=0}^{\infty} \frac{1}{k !}\left(\frac{x}{4 \theta}\right)^{2 k+1} \Gamma_{k+1}(\theta) .
$$

These series converge uniformly since

$$
\Gamma_{i}(\theta)<1 .
$$

However, the usual asymptotic expansion of these functions also has an interest for us here because when $\theta$ is small the convergence is slow and the accuracy is not good. For this development we write Psi as

$$
\psi(x, \theta)=\frac{1}{\sqrt{ } \pi} \int_{-\infty}^{\infty} \frac{e^{-u^{2}}}{1+(u \sqrt{ }(4 \theta+x))^{2}} d u .
$$

By substituting the expansion

$$
\frac{1}{1+(u \sqrt{ }(4 \theta+x))^{2}}=\frac{1}{2} \sum_{n=0}^{\infty}\left[\frac{(-i)^{n}}{(1+i x)^{n+1}}+\frac{(i)^{n}}{(1-i x)^{n+1}}\right](u \sqrt{ }(4 \theta))^{n}
$$

in the equation above and then integrating term by term one obtains 


$$
\begin{aligned}
& \psi(x, \theta)=\frac{2}{\sqrt{ } \pi} \sum_{n=0}^{\infty} \frac{(-4 \theta)^{n}}{\left(\sqrt{ }\left(1+x^{2}\right)\right)^{2 n+1}} \\
& \cdot \cos \left((2 n+1) \cos ^{-1}\left(x^{2}+1\right)^{-1 / 2}\right) \int_{0}^{\infty} u^{2 n} e^{-u^{2}} d u
\end{aligned}
$$

where the following identity was employed

$$
(1+i x)^{-2 n-1}+(1-i x)^{-2 n-1}=2\left(1+x^{2}\right)^{n+1 / 2} \cos \left((2 n+1) \cos ^{-1}\left(1+x^{2}\right)^{-1 / 2}\right) \text {. }
$$

Since

$$
\int_{0}^{\infty} u^{2 n} e^{-u^{2}} d u=\frac{|1 \cdot 3 \cdot 5 \cdots(2 n-1)|}{2^{n+1}} \sqrt{ } \pi
$$

and

$$
\cos \left((2 n+1) \cos ^{-1}\left(x^{2}+1\right)^{-1 / 2}\right)=T_{2 n+1}\left(\left(1+x^{2}\right)^{-1 / 2}\right)
$$

where

$$
T_{k}(x) \text { is a Tchebycheff polynomial }
$$

defined by

$$
T_{0}(x)=1, T_{1}(x)=x
$$

and

$$
T_{k+1}(x)=2 x T_{k}(x)-T_{k-1}(x),
$$

we have

$$
\psi(x, \theta)=\sum_{n=0}^{\infty} \frac{|1 \cdot 3 \cdot 5 \cdots(2 n-1)|(-2 \theta)^{n}}{\left(\sqrt{ }\left(x^{2}+1\right)\right)^{2 n+1}} T_{2 n+1}\left(1 / \sqrt{ }\left(x^{2}+1\right)\right) .
$$

Expansion (2) is permissible because of Watson's lemma [2, p. 236]. Similarly

$$
\chi(x, \theta)=\sum_{n=0}^{\infty} \frac{|1 \cdot 3 \cdot 5 \cdots(2 n-1)|(-2 \theta)^{n}}{\left(\sqrt{ }\left(x^{2}+1\right)\right)^{2 n+1}} T_{2 n+1}\left(x / \sqrt{ }\left(x^{2}+1\right)\right) .
$$

United Technology Center

Sunnyvale, California

1. G. Goertzel, "The 1)oppler effect in intermediate and fast reactions," Proceedings of the International Conference of Peaceful Uses of Atomic Energy, Vol. 5, 1956, p. 472.

2. G. N. Watson, A Treatise on the Theory of Bessel Functions, 2nd ed., Cambridge Univ. Press, New York, and Macmillan, New York, 1958, 804 pp.

3. D. G. Hummer, and Macmillan, New York, "Expansion of Dawson's function in a series of Chebyshev polynomials, Math. of Comp., v. 18, 1964, pp. 317-319. 